\title{
A Hybrid Method for Face Recognition using LLS CLAHE Method
}

\author{
A. Thamizharasi \\ Assistant Professor, \\ Department of Computer \\ Science \& Engineering, \\ Mohandas College of Engineering and \\ Technology, \\ Anad, Nedumangad P.O., \\ Trivandrum, Kerala, India
}

\author{
Jayasudha J. S., PhD \\ Professor, \\ Department of Computer \\ Science \& Engineering, \\ SCT College of Engineering, \\ Pappanamcode, \\ Trivandrum, Kerala, India
}

\begin{abstract}
Face recognition is an active research work since its use is widespread in many applications. The proposed work is to develop a hybrid illumination pre-processing method for face recognition by combining two-dimensional Discrete Wavelet Transform (2D DWT) and Contrast Limited Adaptive Histogram Equalization (CLAHE). 2D DWT is applied on the original image and the LL sub-band of DWT coefficients is extracted. These coefficients are multiplied by a scaling factor and then CLAHE is applied on it. The image obtained is termed as LLS CLAHE. The face recognition of LLS CLAHE is done using Gabor fisher classifier method. The efficiency of the proposed method is tested on AR, Yale and ORL, Extended Yale B and CMU PIE databases. The experimental results prove that LLS CLAHE using Gabor fisher is an effective method for images under varying illuminations.
\end{abstract}

\section{General Terms}

face recognition, 2D DWT, CLAHE, LLS CLAHE, illumination pre-processing, Gabor Fisher.

\section{Keywords}

illumination pre-processing, face recognition.

\section{INTRODUCTION}

Face recognition is nowadays widespread in many applications like security and surveillance systems, social networks, identification systems etc. Automated face recognition is a huge research area and many researchers attempted each year to improve the recognition accuracy. Till now, it is an active research area due to its complexity. A face image can be recognized from still image or video. The face recognition task by itself is a complex process and further complicated due to added factors like variations in illuminations, facial expressions, pose changes, aging etc. Among the various challenges variations in illumination affects the recognition accuracy worse.

The feature based methods uses the extracted features or feature descriptors for face recognition. One of the feature based methods, Discrete Wavelet Transform (DWT) is a multi-resolution technique useful in biometrics for edge detection and feature extraction. DWT analyses the signal in frequency domain and time domain simultaneously. The signal to be analyzed is passed through low pass filters and high pass filters at different levels. At each level it extracts low-frequency components (or approximation coefficients) and high-frequency components (or detail coefficients) [1].
2D DWT when applied at level one, results in four decomposed sub-bands or sub-images. The four sub-bands are represented as Low-Low (LL), Low-High (LH), High-Low (HL) and High-High (HH). These four sub-bands are also denoted as $\mathrm{L} 1, \mathrm{H} 1, \mathrm{~V} 1$ and $\mathrm{D} 1$ respectively [2]. In the 2D DWT, image of size $\mathrm{m} \times \mathrm{n}$ is divided into four sub-bands L1, H1, V1 and D1 also called as approximation coefficients, horizontal coefficients, vertical coefficients and diagonal coefficients respectively.

The wavelet coefficients have much redundant or irrelevant information [1]. DWT could able to extract coarse to fine facial features. The approximation coefficients are illumination sensitive components and detail coefficients are illumination insensitive components. But, detail coefficients affect the facial expression. The facial variations are confined mostly to local regions [1]. The Contrast Limited Adaptive Histogram Equalization (CLAHE) is a contrast enhancement method that uses the concepts of AHE and CLHE [3].

The objective of this research work is to develop hybrid illumination pre-processing methods for recognizing the frontal face images with varying illuminations, changes in facial expressions and images under difficult light conditions. The authors developed a novel illumination normalization method termed LLS CLAHE for poor illumination images [4]. The face image in this work is classified using Fuzzy K Nearest Neighbour classifier. The proposed hybrid method is developed by combining LLS CLAHE and Gabor Fisher classifier.

\section{RELATED WORKS}

Etemad et al. (1997) proposed discriminant analysis on wavelet coefficients [5]. Garcia et al. (2000) proposed wavelet packet decomposition method for frontal face recognition under roughly constant illumination. Each face image is described by a subset of band filtered images containing wavelet coefficients. Feature vectors are created from these wavelet coefficients using simple statistical measures [6]. Chien \& Wu (2002) proposed wavelet based approach called Waveletface [7]. It uses the wavelet transform to decompose the image data into four sub images. Linear Discriminant Analysis is applied to increase the discriminating power.

Zhang et al. (2009) proposed Multiscale Facial Structure Representation which can reduce the effect of illumination by wavelet-based denoising techniques and soft threshold [8]. Harin Sellahewa et al. (2010) proposed a quality based adaptive approach that does fusion of match scores from lowfrequency and high-frequency based face representations for 
improving recognition accuracy under varying lighting conditions [9].

Hafiz Imtiaz and Shaikh Anowarul Fattah (2011) developed a wavelet-based face recognition scheme. Instead of the entire face image, this scheme considers only some high-informative local zones of the image for dominant feature extraction [10]. Cao et al. (2012) proposed an illumination invariant wavelet based approach that considers the correlation of neighboring wavelet coefficients [11].

Wenkai Xu et al. (2014) presented an integrated algorithm for face recognition by combining Wavelets Transform (WT), 2D Principal Component Analysis (PCA) and Support Vector Machines (SVM) [12]. Huang et al. (2015) presented a novel image enhancement method, named 'CLAHE-DWT' by combining CLAHE and DWT [13]. This method included three main steps. First, the original image is decomposed into low-frequency and high-frequency components by DWT. Then, the low-frequency components are enhanced using CLAHE and the high-frequency components are unchanged. Finally, image is reconstructed by taking the inverse DWT of the modified low-frequency and high-frequency components.

The DWT based illumination normalization methods does not requires models and could represent the facial features from coarse to fine details. These methods have low computational complexity and make it suitable for real world applications.

Štruc V et al. (2010) developed face recognition technique called Complete Gabor Fisher Classifier (CGFC) [14]. The Complete Gabor Fisher Classifier (CGFC) created a framework that combines Gabor magnitude information and features computed based on Gabor phase information [14]. The Gabor Wavelet is a powerful tool for facial feature extraction and robust face recognition. Štruc V et al. computes the matching scores using both Gabor magnitude vector and Gabor phase information. A decision level fusion is applied to the matching scores of the two feature vectors.

\section{METHODOLOGY}

\subsection{Algorithm for face recognition of LLS CLAHE using Gabor Fisher classifier method}

The algorithm for face recognition of LLS CLAHE using Gabor Fisher classifier method is given below.

Step 1: Read the input image

Step 2: Apply Discrete Wavelet Transform to extract the four sub-bands LL, LH, HL and HH

Step 3: LL sub-band is multiplied by scalar value 1.1.

Step 4: The Inverse DWT is applied to the modified LL coefficients and the unchanged edge coefficients

Step 5: Apply CLAHE for creating LL S CLAHE image.

Step 6: Create training database and testing database by applying steps 1 to 4 to the original image.

Step 7: Apply face recognition algorithm using Gabor Fisher classifier

Step 8: Compute rank one recognition rate and verification rate at $1 \%$ False Acceptance Rate percentages.

2D Discrete Wavelet Transform (DWT) using haar wavelet is applied on the original image and approximation coefficients are extracted. These coefficients are multiplied by scalar value
1.1. The inverse DWT is applied using the modified approximation coefficients and unchanged detail coefficients; Then CLAHE is applied on the image. The output image obtained is termed as 'LLS CLAHE' image as LL sub-band coefficients are multiplied by a scalar value.

In this work, the Gabor Wavelet is applied with five scales ( 0 to 4) in eight orientations (zero to seven) and Gabor magnitude is extracted. This process creates a Gabor magnitude feature vector. The Gabor magnitude responses are down sampled by 64 to reduce the dimensionality and then it is normalized to zero mean and unit variance. The Gabor Fisher Classifier is obtained by applying the fisherfaces algorithm to normalized Gabor magnitude feature vector. This work does not use decision level fusion for matching the scores.

\subsection{Description of Databases}

The AR (Alianz Martinez and Robert Benavente) database contains images of 100 persons taken in two different sessions [15]. The images are neutral, expressions (anger, scream, and smile), illumination (right, left and both sides) and occlusions. There are 26 images for each person and totally there are 2600 images. The occluded face images are not included for testing purpose. Thus the AR face image subset is created with 14 images of each person with a total of 1400 images. During testing, setting the training to testing ratio as 2:12 means first two images of person were chosen as training images per person while the remaining twelve images per person were chosen as test images.

The Yale Face Database contains 165 gray scale images of 15 individuals in GIF format. There are eleven images per individual. The variations in images are facial expression, center-light, with and without glasses, happy, left-light/no glasses, normal, right-light, sad and sleepy, surprised and wink [16].

The Olivetti Research Laboratory (ORL) face database contains ten different images of each of 40 distinct subjects and each image is of size $112 \times 92$ [17]. The images were taken at different times and it has variations in lighting, facial expressions and head pose variations. All the images were taken in dark homogeneous background.

The Extended Yale B database consists of 28 subjects with 16,128 images [18]. This database contains gray-scale images of 28 subjects each in 9 different poses and 64 different illumination conditions. The experiments are done using only frontal face images. The frontal faces images which contain 28 subjects each in 64 illumination conditions with a total of 1792 images are used for testing.

Carnegie Mellon University Pose Illumination Expression (CMU PIE) database contains 41,368 color images (each of size $486 \times 640$ pixels) of 68 individuals [19]. They are captured under various poses, illuminations and expressions. Each subject in PIE database is in twenty one different illumination conditions with background light off condition. Thus a total of 1428 images (68 subject's $\times 21$ images) are used for testing.

\subsection{Experimental results for LLS CLAHE method}

The experimental work is implemented using MATLAB [20]. The sample output images obtained using the algorithm in Extended Yale B database is shown in figures 1a-e. The sample images in various databases are shown in figure 2 . 

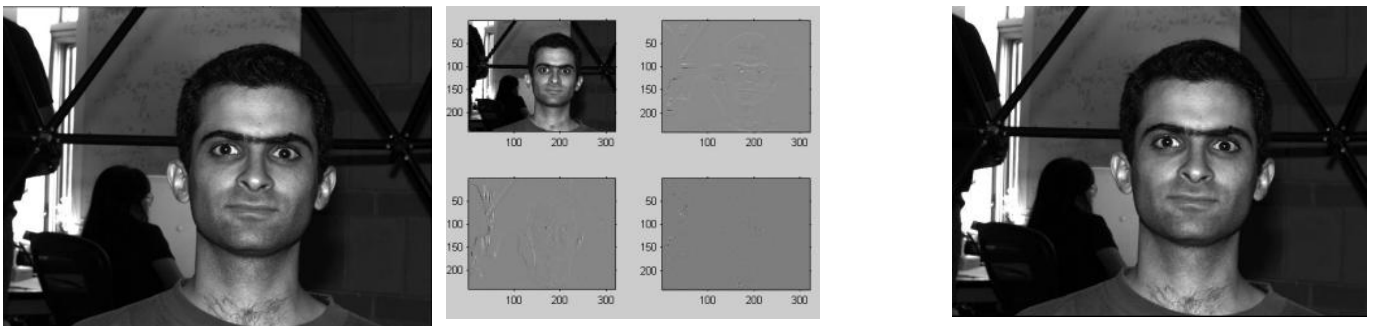

Figure 1a.Original Image

Figure 1b. Four sub-bands of DWT

Figure 1c. LL sub-band Scaled

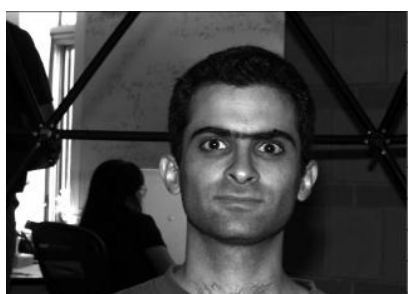

Figure 1d. Inverse DWT of LLS

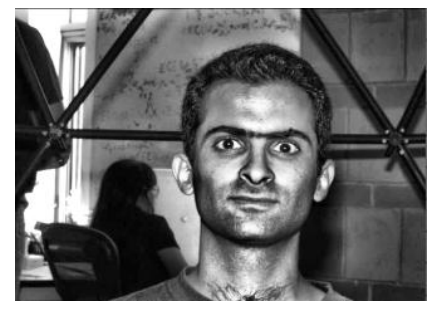

Figure 1e. LLS CLAHE image

Figure 1.Sample Images of LLS CLAHE

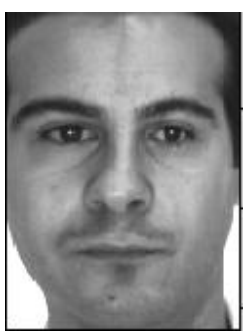

a. Original image in AR database

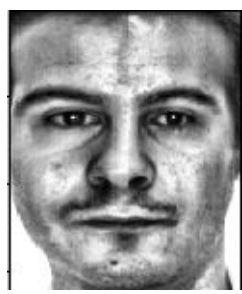

$$
\begin{gathered}
\text { e. LLS CLAHE } \\
\text { image in AR } \\
\text { database }
\end{gathered}
$$
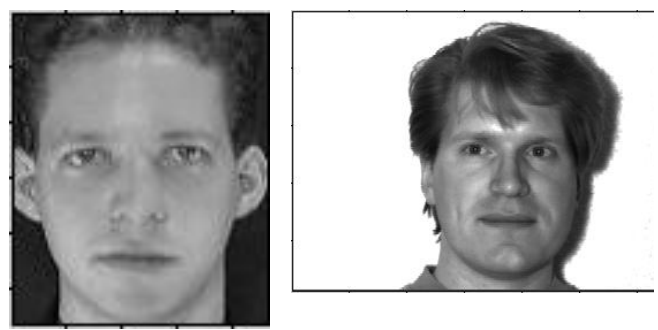

b. Original image in ORL database

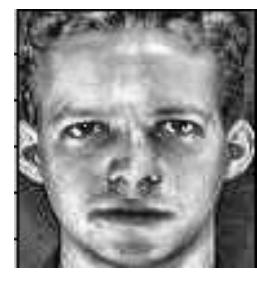

$$
\begin{gathered}
\text { f. LLS CLAHE } \\
\text { image in ORL } \\
\text { database }
\end{gathered}
$$

c. Original image in Yale database

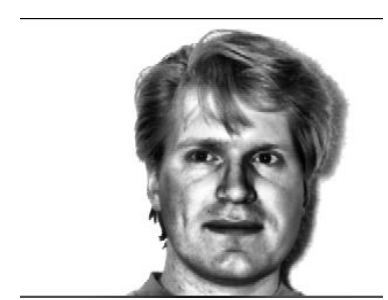

g. LLS CLAHE image in Yale database

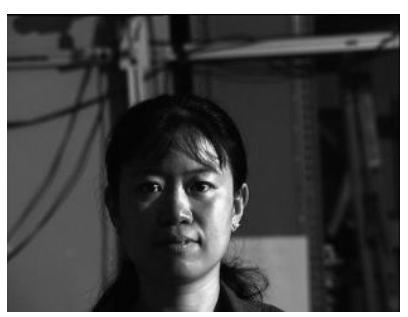

d. Original image in CMU PIE database

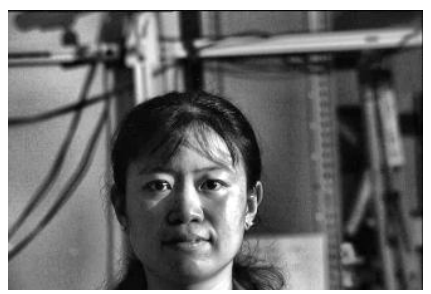

h. LLS CLAHE image in CMUPIE database

Figure.2 Sample Input and Output images of LLS CLAHE in various databases

\subsection{Performance Analysis}

The performance for face recognition of LLS CLAHE using Gabor Fisher is shown in Table 1. The experimental work is carried out on AR database using training to testing ratio of 2:12 and 5:9. The testing is done on Yale database using training to testing ratio of $2: 9$ and $3: 8$. On ORL database, the training to testing ratios used is $2: 8$ and 5:5. The training to testing ratio of Extended Yale B and CMU PIE databases is 2:62 and 2:19 respectively. The recognition accuracy rate percentage is computed for all the databases.
Table 1. Performance Analysis of LLS CLAHE using Gabor Fisher

\begin{tabular}{|l|c|c|}
\hline Database & $\begin{array}{l}\text { Training } \\
\text { to testing } \\
\text { ratio }\end{array}$ & $\begin{array}{l}\text { Recognition } \\
\text { Accuracy } \\
\text { rate (\%) }\end{array}$ \\
\hline AR & $2: 12$ & 93.92 \\
\cline { 2 - 3 } & $5: 9$ & 98.56 \\
\hline Yale & $2: 9$ & 97.78 \\
\cline { 2 - 3 } & $3: 8$ & 100 \\
\hline
\end{tabular}




\begin{tabular}{|l|c|c|}
\hline \multirow{2}{*}{ ORL } & $2: 8$ & 90.31 \\
\cline { 2 - 3 } & $5: 5$ & 98.50 \\
\hline Extended Yale B & $2: 62$ & 97.93 \\
\hline CMU PIE & $2: 19$ & 89.78 \\
\hline
\end{tabular}

Table 2. Performance comparison of LLS CLAHE on ORL database

\begin{tabular}{|c|l|c|}
\hline $\begin{array}{l}\text { Serial } \\
\text { No. }\end{array}$ & Method & $\begin{array}{l}\text { Recognition } \\
\text { Accuracy } \\
\text { Rate \% }\end{array}$ \\
\hline 1 & LDA [21] & 82.00 \\
\hline 2 & U-LDA [21] & 85.00 \\
\hline 3 & PCA [21] & 88.50 \\
\hline 4 & R-LDA [21] & 88.50 \\
\hline 5 & D-LDA [21] & 89.50 \\
\hline 6 & KPCA [22] & 90.50 \\
\hline 7 & HE+DWT [23] & 90.66 \\
\hline 8 & INUM+DWT (3:7) [23] & 90.75 \\
\hline 9 & N-LDA [21] & 91.00 \\
\hline 10 & O-LDA [21] & 91.00 \\
\hline 11 & Complete 2DPCA [22] & 91.50 \\
\hline 12 & KPCA [24] & 91.75 \\
\hline 13 & HE+INUM+DWT [23] & 92.16 \\
\hline 14 & 2DLDA [21] & 92.50 \\
\hline 15 & DWT 1D [23] & 92.66 \\
\hline 16 & PCA+LDA [21] & 93.00 \\
\hline 17 & ASDCT (5:5) [25] & 93.40 \\
\hline 19 & MPCA [22] & 94.44 \\
\hline 20 & 2D PCA [26] & 96.00 \\
\hline 21 & K2DPCA [22] & 96.20 \\
\hline 22 & SVM [27] & 96.40 \\
\hline 23 & DWT+2DPCA+SVM & 97.10 \\
\hline 24 & KSPCA [22] & 97.70 \\
\hline 25 & Kernel Eigenfaces (5:5) & 98.00 \\
\hline 26 & $\begin{array}{l}\text { LLS CLAHE using } \\
\text { Gabor Fisher }\end{array}$ \\
\hline & $\mathbf{9 8 . 5 0}$ \\
\hline
\end{tabular}

LLS CLAHE using Gabor Fisher shows recognition accuracy of $98.56 \%$ and $97.93 \%$ in AR and Extended Yale B database respectively. The performance of LLS CLAHE method on ORL and Yale databases are compared with the results shown in Table 2 and Table 3 respectively.

From table 2, it is found that the results of LLS CLAHE shows recognition accuracy rate of $98.5 \%$ and outperforms the methods under comparison. LLS CLAHE using Gabor Fisher is better than PCA, LDA based methods and kernel methods.

Table 3. Performance Comparison of LLS CLAHE on Yale database

\begin{tabular}{|c|l|c|}
\hline $\begin{array}{l}\text { Serial } \\
\text { No. }\end{array}$ & Method & $\begin{array}{l}\text { Recognition } \\
\text { Accuracy } \\
\text { Rate \% }\end{array}$ \\
\hline 1 & MPCA [22] & 94.44 \\
\hline 2 & 2DPCA(5:6) [22] & 90.08 \\
\hline 3 & KPCA (5:6) [22] & 89.00 \\
\hline 4 & KSPCA [22] & 95.00 \\
\hline 5 & $\begin{array}{l}\text { Kernel Eigenfaces P=3 } \\
(6: 5)[28]\end{array}$ & 75.76 \\
\hline
\end{tabular}

\begin{tabular}{|c|l|c|}
\hline 6 & $\begin{array}{l}\text { Kernel fisherfaces } \\
\text { P=3,G (6:5) [28] }\end{array}$ & 93.94 \\
\hline 7 & K2DPCA (5:6) [22] & 98.00 \\
\hline 8 & 2DPCA (7:4) [27] & 88.60 \\
\hline 9 & SVM (7:4) [27] & 93.20 \\
\hline 10 & $\begin{array}{l}\text { DWT+2DPCA+SVM } \\
(7: 4)[27]\end{array}$ & 94.30 \\
\hline 11 & LDA (6:5) [21] & 88.00 \\
\hline 12 & PCA (6:5) [21] & 81.00 \\
\hline 13 & PCA+LDA (6:5) [21] & 92.22 \\
\hline 14 & D-LDA(6:5) [21] & 87.62 \\
\hline 15 & N-LDA(6:5) [21] & 94.29 \\
\hline 16 & O-LDA(6:5) [21] & 94.29 \\
\hline 17 & U-LDA(6:5) [21] & 96.00 \\
\hline 18 & R-LDA(6:5) (6:5) [21] & 91.43 \\
\hline 19 & 2DLDA(6:5) [21] & 78.67 \\
\hline 20 & PCA [25] & 83.99 \\
\hline 21 & LDA [25] & 90.10 \\
\hline 22 & KFA [25] & 76.88 \\
\hline 23 & KPCA [25] & 68.82 \\
\hline 24 & ASDCT [25] & 95.95 \\
\hline 25 & LLS CLAHE using & $\mathbf{1 0 0}$ \\
\hline
\end{tabular}

Table 3 shows that LLS CLAHE using Gabor Fisher method outperforms twenty five existing methods on Yale database using training to testing ratio of 3:8 and shows recognition accuracy rate of $100 \%$. LLS CLAHE using Gabor Fisher is better than PCA, LDA based methods, kernel methods and Discrete Cosine Transform based methods.

The performance of LLS CLAHE method on outperforms PCA, LDA, KFA, KPCA and ASDCT methods on Extended Yale B database [25]. The results of LLS CLAHE using Gabor Fisher shows that it is robust under pose variations, scale changes, facial expressions and varying illuminations.

\section{CONCLUSION}

The illumination normalization of face images is done using 2D DWT approximation coefficients and CLAHE. The performance results of LLS CLAHE using Gabor Fisher in ORL, Yale, AR and Extended Yale B shows that it is robust to illumination variations, pose variations, scale changes, facial expressions and varying illuminations.

The face recognition work can be extended by tagging the label name to the face which can be used for face annotation applications. The person identification from videos is another emerging application which uses gestures for identification. This work can also be extended to identify the facial expressions for developing an automatic emotion analysis system.

\section{ACKNOWLEDGMENTS}

The authors would like to thank the friends and the reviewers.

\section{REFERENCES}

[1] Dao Qing Dai and Hong Yan, Wavelets and Face Recognition.: ISBN 978-3-902613-03-5, I-Tech, Austria, pp. 558, 2007

[2] Jamie Cook, Vinod Chandran and Sridha Sridharan , "Multi scale representation for 3-D Face Recognition," IEEE Transactions on Information Forensics and Security, Vol. 2, No. 3, pp. 529-536, 2007.

[3] Zuiderveld K., "Contrast Limited Adaptive Histogram Equalization", Academic Press, Cambridge, MA, 1994 
[4] A.Thamizharasi \& Dr.Jayasudha J.S, "A Novel Illumination Normalization technique for enhancing face images with uneven illumination", IETE International Conference on Robotics and Automation (ICRA 2016), February, 2016. (Article communicated to IETE Journal of Research for publication)

[5] Etemad K. and Chellappa R., "Discriminant Analysis for recognition of human face images," Journal of Optical Society America, Vol. 14, No. 8, 1997

[6] C.Garcia , G.Zikos and G.Tziritas, "Wavelet packet analysis for face recognition," Image and Vision Computing, Vol. 18, pp. 289-297, 2000

[7] J.T.Chien and C.C.Wu, "Discriminant Waveletfaces and nearest feature classifiers for face recognition," IEEE Transactions on Pattern Analysis and Machine Intelligence, Vol. 24, pp. 1644-1649, 2002

[8] T.P.Zhang, B.Fang, Y.Yuan, Y.Y.Tang and Z.W.Shang, "Multiscale facial structure representation for face recognition under varying illumination," Pattern Recognition, Vol. 42, pp. 251-258, 2009

[9] Harin Sellahhewa and Sabah A.Jassim , "Image Quality Based Adaptive Face Recognition," IEEE Transactions on Instrumentation and Measurement, Vol. 59, No. 4, 2010

[10] Hafiz Imtiaz and Shaikh Anowarul Fattah, "A Face Recognition Scheme using Wavelet Based Dominant Features," Signal and Image Processing: An International Journal, Vol. 2, No. 3, 2011

[11] Cao X., W.Shen, L.G.Yu, Y.L.Wang and Z.W.Zhang, "Illumination invariant extraction for face recognition using Neighboring Wavelet Coefficients," Pattern Recognition, Vol. 45, pp. 1299-1305, 2012

[12] Wenkai Xu and Eung Joo Lee, "Face Recognition Using Wavelets Transform and 2D PCA by SVM Classifier," International Journal of Multimedia and Ubiquitous Engineering, Vol. 9, No. 3, pp. 281-290, 2014

[13] Huang Lidong, Zhao Wei, Wang Jun and Sun Zebin, "Combination of contrast limited adaptive histogram equalization and discrete wavelet transform for image enhancement," IET Image Processing, Vol. 9, No. 10, pp. 908-915, 2015

[14] Struc V. and Pavesic N., "The Complete Gabor-Fisher Classifier for Robust Face Recognition," EURASIP Advances in Signal Processing, Vol. 1155, Pages.26, 2010

[15] A.M.Martinez and R.Benavente, "The AR Face Database," 24, CVC Technical Report, 1998.

[16] P.N.Belhumeur, J.P.Hespanha and D.J Kriegman, "Eigenfaces vs Fisherfaces: Recognition using class specific linear projection," IEEE Transactions on Pattern Analysis and Machine Intelligence, Vol. 19, No. 7, 1997.

[17] ORL Face database. [Online]. http://www.cl.cam.ac.uk/research/dtg/attarchive/facedata base.html (Checked on 10th September 2016)

[18] K.C.Lee, J.Ho and D.Kriegman, "Acquiring Linear Subspaces for Face Recognition under Variable Lighting," IEEE Transactions on Pattern Analysis and Machine Intelligence, vol. 27, No. 5, pp. 684-698, 2005
[19] T.Sim, S.Baker and M.Bsat, "The CMU Pose, Illlumination and Expression Database," IEEE Transactions on Pattern Analysis and Machine Intelligence, Vol. 25, No. 12, 2003

[20] Rafael C.Gonzalez , Richard E.Woods and Steven L.Eddins, "Digital Image processing with MATLAB", 2nd Edition, 2009

[21] Kai Li, Zhen Liu and Peng Tang , "On Linear Discriminant Analysis and its Variants in Face Recognition," International Journal of Artificial Intelligence and Mechatronics, Vol. 4, No. 1, 2015.

[22] Jamal Husain Shah , Muhammad Sharif, Mudassar Raza and Aisha Azeem, "A Survey: Linear and Nonlinear PCA Based Face Recognition Techniques," The International Arab Journal of Information Technology, Vol. 10, No. 6, 2013.

[23] Gautham Sitaram Yajia, Sankhadeep Sarkara, K Manikantana and S Ramachandran, "DWT feature extraction based face recognition using intensity mapped unsharp masking and laplacian of Gaussian filtering with scalar multiplier," in Second International Conference on Communication, Computing and Security (ICCCS2012), Procedia Technology, Vol.6, pp. 475-484, 2012

[24] Liu N., Wang H. and Yau W., "Face Recognition with Weighted Kernel Principal Component Analysis," in Proceedings of the nineth International Conference on Control, Automation, Roboticcs and Vision, pp. 1-5, 2006.

[25] Zahraddeen Sufyanu, Fatma S.Mohamad, Abdulganiyu A.Yusuf and Mustafa B.Mamat, "Enhanced Face Recognition Using Discrete Cosine Transform," Engineering Letters, Vol. 24, No. 1, pp. 52-61, 2016

[26] Jian Yang, David Zhang, Alexandro F Frangi and Jing Yu Yang, "Two Dimensional PCA: A New Approach to Appearance-based Representation and Recognition," IEEE Transactions on Pattern Analysis and Machine Intelligence, Vol. 26, No. 1, pp. 131-137, 2004

[27] Wenkai Xu and Eung Joo Lee, "Face Recognition Using Wavelets Transform and 2D PCA by SVM Classifier," International Journal of Multimedia and Ubiquitous Engineering, Vol. 9, No. 3, pp. 281-290, 2014

[28] M.H Yang, "Kernel Eigenfaces vs Kernel Fisherfaces: Face Recognition using Kernel Methods," in Proceedings of fifth IEEE International Conference on Automatic Face and Gesture Recognition (RGR'02), pp. 215-220, 2002

[29] Taiping Zhang, Yuan Yan Tang, Bin Fang and Xiaoyu Liu, "Face Recognition Under Varying Illumination using Gradientfaces," IEEE Transactions on Image Processing, Vol. 18, No. 11, pp. 2599-2606, 2009 